\title{
Translating Output Specifications to Production Flow Requirements for Re-entrant Lines*
}

\author{
Ming-Der Hu, Shi-Chung Chang \\ Department of Electrical Engineering \\ National Taiwan University \\ Taipei, Taiwan, R.O.C. \\ joseph@ac.ee.ntu.edu.tw, scchang@ac.ee.ntu.edu.tw
}

\begin{abstract}
A backward analysis (BA) algorithm is designed in this paper to translate the desired output specifications of a reentrant production line into internal flow requirements for machine groups. These requirements may serve as guidelines for distributed production flow control. Specific output specifications considered are the mean and variance of inter-departure time of the line output process, while production flow requirements are the target mean rates and upper bounds on squared coefficient of variation of individual machine input processes. Our BA is developed on top of an open queueing network (OQN) model of re-entrant lines. It combines decompositionbased queueing network analysis techniques to approximate, for a re-entrant $\mathrm{OQN}$, the relationship between the characteristics of the output process and the characteristics of external input and machine service processes. The BA then derives, as two sets of linear equations, input flow requirements for machine groups from line output specifications and machine service characteristics. Preliminary simulation study demonstrates that our BA algorithm is reasonably accurate and fast and can be applied to deriving practically measurable production flow requirements.
\end{abstract}

\section{Introduction}

How delivery specifications for a factory should be translated into performance requirements of individual production entities or processing steps within the factory has long been a significant research topic of production control [14]. A modern factory is often operated under distributed control. When shop floor performance requirements are closely mapped to delivery specifications, individual controllers may then operate locally to meet their local performance requirements while effectively achieving the overall specifications. The translation problem has been more challenging than before because the competition for quick, quality and low-cost responses to customer demands in the manufacturing industry is getting fierce worldwide, and manufacturing systems are getting more complex then ever.

* This work was supported in part by the National Science Council of the Republic of China under Grants NSC 86-2622-E-002-025R and NSC 87-2212-E-002-023.

$0-7803-4394-8 / 98 \$ 10.00$ (c) 1998 IEEE
Specifically, semiconductor fabrication is a business characterized by high capital investment, short product life cycle, high product add-on values and stringent customers' demands for both excellent quality and timely delivery. A wafer fabrication plant (fab) involves one of the most complex manufacturing processes. There are several hundreds of operation steps in a process, which involves tens of delicate and expensive equipment groups. A process flow is highly re-entrant because its wafers make multiple visits to an equipment group as successive circuit layers are added onto the wafers. This re-entrant feature poses a unique challenge to production flow control, since wafers of different types as well as wafers of the same type but at different layers of fabrication may compete for the finite capacity of an equipment group. In addition, factors such as scrap, wafer lot split/merge, equipment breakdowns, preventive maintenance, operator availability and setup requirements combine to make the manufacturing environment highly dynamic and uncertain. The translation of delivery specifications to production performance requirements within such re-entrant lines has therefore been a significant research challenge.

In the literature, there have been a few performance models of re-entrant lines including (1) discrete event simulation models [5-6], (2) stochastic optimal control models [7, 9], and (3) queueing network models [3-4]. Among them, a stochastic optimal control model usually leads to analysis of very small problems, although theoretical insights can be generated from it. Discrete event simulation models are usually high-fidelity representations of a real re-entrant line and are frequently used for detailed design evaluation. However, they require quite an amount of development and maintenance efforts and are mostly suitable for descriptive/evaluative rather than prescriptive purposes. Analyses based-on queueing network models has been successfully applied to both planning and performance evaluation problems of realistic scale.

Manufacturing systems are often modeled as open queueing networks (OQNs) and OQN models can be analyzed using either exact methods or approximate methods. Exact methods exist only for a small class of 
product-form OQNs, which is not suitable for modeling realistic re-entrant lines. The most frequently used approximation methods to analyze OQN have been decomposition methods. Associated with renewal-process approximations, decomposition method uses two parameters to characterize the stochastic arrival, service and departure processes in the network, one describing the average and the other describing the variability. There have been quite an amount of research results in this direction $[1,2,4,13,16]$. Specifically, approximation techniques for re-entrant OQNs have been proposed by [1, $4,13]$. Connors et. al. [4] reported a successful application to capacity planning for semiconductor fabs.

In this paper, we consider the mean rate and squared coefficient of variation (SCV) of inter-departure time as specification items of the output process of a re-entrant line. Such a selection is motivated by the fact that the aspect of variability, i.e., the second order statistics, as well as mean values has been brought into production flow control in semiconductor manufacturing $[8,11-12,15]$. We develop a method of translating these specifications to target mean rates and upper bounds on SCVs of individual machine input processes, which may facilitate effective distributed production flow controls. An OQN model for re-entrant lines is first established. Combining results of decomposition-based approximate queueing network analysis, we develop a forward analysis (FA) of the reentrant $O Q N$. The FA derives characteristic parameters of machine output processes from input and machine service processes. Due to the re-entrant feature, the inter-arrival time SCV of the external input process explicitly appears as a term of the inter-departure time SCV of the line output. We then design a backward analysis (BA) algorithm by reversing the $\mathrm{FA}$, where the target mean rates and upper bounds on SCVs of machine input processes are derived as two sets of linear equations using parameters of output/departure and machine service processes. Preliminary simulation study indicates that our BA algorithm has a good potential for quick translation of output specifications to requirements of measurable production performance metrics such as mean cycle time and cycle time variance.

The remainder of the paper is organized as follows. Section II presents the OQN model of a re-entrant line. The FA of the re-entrant OQN model is developed in Section III. Section IV then describes our design of the BA algorithm. Preliminary validation results by simulation are then given in Section V. Finally, concluding remarks are made in Section VI.

\section{Modeling a Re-entrant Line}

Consider a re-entrant line modeled as an OQN illustrated in Figure 1 , where there are $M$ machine groups, $I$ product types and $J$ different production steps. In the re-entrant OQN model each node represents a machine group. Each machine group $m$ has $M_{m}$ identical parallel servers with independent and identically distributed (iid) service times. The inter-arrival times for each product type arrived at the entry node, machine group 1 , are assumed iid. The processing of a production step $j(j=1,2, \ldots, J)$ requires a specific machine group $m_{j}\left(m_{j}=1,2, \ldots, M\right)$ and there is a buffer $b_{j}$ for each operation step. Process flow of each product type $i(i=1,2, \ldots, I)$ is defined by a deterministic sequence of operation steps which is a subsequence of $\{l$, $2, \ldots, \Omega$. A product may visit a machine group more than once, but for simplicity we shall not allow two successive stages of a product route to be identical. Furthermore, it is assumed that the arrival processes and service processes are general distributions, buffers are infinite and work dispatching rule for each machine group is first-come firstserve (FCFS). Also, we restrict our discussion to the longrun average behavior of equilibrium networks.

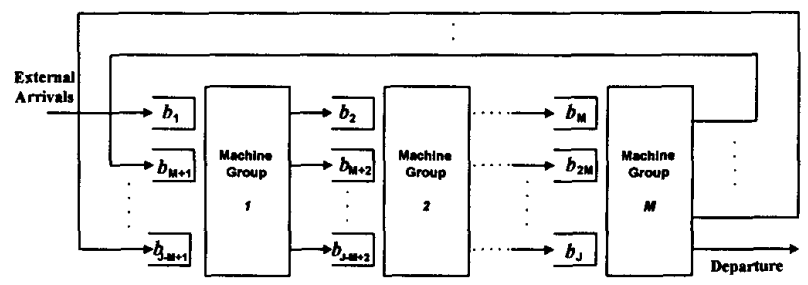

Figure 1: Structure of a Re-entrant Line

\section{Forward Analysis of Re-entrant Lines}

In this section, we follow the general approach of [16] to develop a performance analysis of a re-entrant line based on its OQN model. In particular, we adopt state-of-art decomposition-based approximation techniques $[1,13,17]$ considering multiple job classes and deterministic routing interference.

A decomposition-based approximation analysis essentially attempts to generalize the notion of independence and product form results for Jackson type networks to more general systems. It relies on two notions:

(a) The nodes can be treated independently as $G I / G / m$ queues.

(b) Two parameters, average rate and square coefficient of variation (SCV), are used to characterize an arrival and a service process at each node.

\section{Single Class Aggregation}

To convey our main ideas and to simplify later presentation, we first aggregate multi-class jobs into a single class and develop the corresponding analysis. Our aggregation procedure follows that of Whitt [16] and is briefly summarized below.

Define 
$\lambda_{e}$ : aggregate mean external arrival rate (to node 1 );

$C_{e}^{2}$ : inter-arrival time SCV of aggregate external arrivals to node 1 ;

$C_{i}^{2}$ : interarrival time SCV of type- $i$ arrivals at node 1;

$\lambda_{m n}$ : aggregate mean flow rate from node $m$ to $n$;

$C_{s_{i k}}^{2}$ : service time $\mathrm{SCV}$ of a part type $i$ at its $k$-th processing step;

$\tau_{m}$ : aggregate mean processing time at node $m$;

$C_{s m}^{2}$ : aggregate service time SCV at node $m$;

$m_{s_{i k}}$ : machine group for processing $k$-th operation step of a part type $i, m_{s_{i k}} \in\{1,2, \ldots, M\}$;

$Q=\left\{q_{m n}\right\}$ : routing matrix, where $q_{m n}$ is the ratio of routing jobs out of node $m$ to node $n$;

$1 H(\bullet)$ : a membership function defined on set $H$, where

$$
\mathbf{1} H(x)= \begin{cases}1, & \text { if } \mathrm{x} \in \mathrm{H} \\ 0, & \text { otherwise }\end{cases}
$$

The aggregate external arrival rates and the flow rates within the network are obtained by adding up average flow rates of individual classes:

$$
\begin{aligned}
& \lambda_{e}=\sum_{i=1}^{I} \lambda_{i}, \\
& \lambda_{m n}=\sum_{i=1}^{I} \sum_{k=1}^{s_{i}} \lambda_{i} 1\left\{(i, k): m_{s_{i k}}=m, m_{s_{i(k+1)}}=n\right\}, \forall m \neq n, \\
& m, n=1,2, \ldots, M .
\end{aligned}
$$

It is then quite straight forward that the ratio of routing from node $m$ to node $n$ can be calculated as:

$$
q_{m n}=\frac{\sum_{i=1}^{I} \sum_{k=1}^{s_{i}-1} \lambda_{i} 1\left\{(i, k): m_{s_{i k}}=m, m_{s_{i(k+1)}}=n\right\}}{\sum_{i=1}^{I} \sum_{k=1}^{S_{i}} \lambda_{i} 1\left\{(i, k): m_{s_{i k}}=m\right\}} .
$$

The inter-arrival time SCV of aggregate external arrivals to node 1 is

$$
C_{e}^{2}=\sum_{i=1}^{I} C_{i}^{2} \lambda_{i} / \sum_{i=1}^{I} \lambda_{l}
$$

The average service time aggregate at node $m$ is then

$$
\tau_{m}=\frac{\sum_{i=1}^{I} \sum_{k=1}^{s_{i}} \lambda_{i} \tau_{s_{i k}} \mathbf{1}\left\{(i, k): m_{s_{i k}}=m\right\}}{\sum_{i=1}^{I} \sum_{k=1}^{s_{i}} \lambda_{i} \mathbf{1}\left\{(i, k): m_{s_{i k}}=m\right\}},
$$

and the corresponding SCV [16] is

$$
C_{s m}^{2}=\frac{\sum_{i=1}^{I} \sum_{k=1}^{s_{i}} \lambda_{i} \tau_{s_{i k}}^{2}\left(C_{s_{i k}}^{2}+1\right) \mathbf{1}\left\{(i, k): m_{s_{i k}}=m\right\}}{\sum_{i=1}^{I} \sum_{k=1}^{s_{i}} \lambda_{i} \mathbf{1}\left\{(i, k): m_{s_{i k}}=m\right\} \tau_{m}^{2}}-1 \text {. }
$$

\section{Forward Analysis}

In the decomposition approach, traffic equations form a critical link in terms of relating the external input traffic characteristics and service node characteristics to the internal traffic flow characteristics of individual nodes.

Denote $\lambda_{a m}:$ mean total arrival rate to node $m$;

$C_{a m}^{2}$ : inter-arrival time SCV at node $m$;

$C_{d m}^{2}:$ inter-departure time SCV at node $m$;

$C_{m n}^{2}$ : inter-departure time SCV for the flow going from node $m$ to node $n$.

When the network is in a steady state, there is a flow rate balance relationship among network nodes,

$$
\begin{aligned}
\lambda_{a 1} & =\lambda_{e}+\sum_{m=1}^{M} \lambda_{m l}, \\
\lambda_{a n} & =\sum_{m=1}^{M} \lambda_{m n} \\
& =\sum_{m=1}^{M} \lambda_{a m} q_{m n}, \quad \text { for } n=2, \ldots, M,
\end{aligned}
$$

which are known as traffic rate equations. In (3.7), there are $M$ equations for the $M$ unknown variables $\left\{\lambda_{a m}, m=1\right.$, $2, \ldots, M\}$. Once the values of $\left\{\lambda_{a m}\right\}$ are obtained by solving (3.7) for a given set of $\left\{\lambda_{i}, i=1,2, \ldots, I\right\}$, the traffic intensity (expected utilization) of node $m$ can be calculated by

$$
\rho_{m}=\frac{\lambda_{a m} \tau_{m}}{M_{m}}
$$

We then examine the relationship among SCVs of the network. Note that the departure flow out of a node is split into a few sub-flows of different destination nodes according to the routing matrix $\left\{q_{m n}\right\}$. However, in a reentrant line, the routing depends not only on part type and machine, but also on the step just completed. Bitran and Tirupati [1] showed that Markovian routing can be a poor approximation for multi-class OQNs with deterministic routings. After simulation study of approximation models for re-entrant queues developed by $[1,13]$, we adopt the approximation formula of [13]. Suppose that $C_{d m}^{2}$ is known for node $m$. The variability parameter $C_{m n}^{2}$ is then refined by

$$
C_{m n}^{2}=q_{m n} C_{d m}^{2}+\left(1-q_{m n}\right) q_{m n} C_{a m}^{2}+\left(1-q_{m n}\right)^{2} C_{e m}^{2},
$$

where $C_{e m}^{2}$ is the average of external arrival process variability parameters, weighted by the expected number of visits of each class at node $m$,

$$
C_{\mathrm{em}}^{2}=\frac{\sum_{i=1}^{I} \sum_{k=1}^{s_{i}} \lambda_{i} \mathbf{1}\left\{(i, k): m_{s_{i k}}=m\right\} C_{i}^{2}}{\sum_{i=1}^{I} \sum_{k=1}^{s_{i}} \lambda_{i} 1\left\{(i, k): m_{s_{i k}}=m\right\}} .
$$

The departure process out of node $m$ is effected by both the arrival and the service characteristics of node $m$. The inter-departure time SCV at node $m$ is approximated by [16]

$$
C_{d m}^{2}=1+\left(1-\rho_{m}^{2}\right)\left(C_{a m}^{2}-1\right)+\frac{\rho_{m}^{2}\left(\max \left\{C_{s m}^{2}, 0.2\right\}-1\right)}{\sqrt{M_{m}}} .
$$


The aggregate arrival process at node $n$ is a superposition of all split departure sub-flows coming from other nodes. Define $\lambda_{o 1}=\lambda_{e}, \lambda_{o n}=0$, for $n=2, \ldots, M, C_{o l}^{2}=C_{e}^{2}$ and $C_{o n}^{2}=0$ for $n=2, \ldots, M$. By utilizing the procedure of [16, Section 4.3], we can obtain the inter-arrival time SCV of an aggregate arrival process as

$$
C_{a n}^{2}=1-\varpi_{n}+\varpi_{n} \sum_{m=0}^{M} \frac{\lambda_{m n}}{\lambda_{a n}} C_{m n}^{2}, n=1,2, \ldots, M,
$$

where $\varpi_{n}=\left[1+4\left(1-\rho_{n}\right)^{2}\left(v_{n}-1\right)\right]^{-1}$,

and $\quad v_{n}=\left[\sum_{m=0}^{M}\left(\lambda_{m n} / \lambda_{a n}\right)^{2}\right]^{-1}$.

Substituting (3.9) into (3.12), we express $C_{a n}^{2}$ as

$$
C_{a n}^{2}=a_{n}+\sum_{m=1}^{M} C_{a m}^{2} b_{m n}, \quad n=1,2, \ldots, M ;
$$

where

$$
\begin{aligned}
& a_{n}=1+\varpi_{n}\left\{\frac{\lambda_{o n}}{\lambda_{a n}} C_{o n}^{2}-1+\sum_{m=1}^{M}\left(\frac{\lambda_{m n}}{\lambda_{a n}}\right)\left[q_{m n} \rho_{m}^{2} \chi_{m}+\left(1-q_{m n}\right)^{2} C_{e n}^{2}\right]\right\}, \\
& b_{m n}=\varpi_{n}\left(\frac{\lambda_{m n}}{\lambda_{a n}}\right) q_{m n}\left[\left(1-\rho_{m}^{2}\right)+\left(1-q_{m n}\right)\right], \\
& \chi_{m}=1+\frac{\left(\max \left\{C_{s m}^{2}, 0.2\right\}-1\right)}{\sqrt{M_{m}}} .
\end{aligned}
$$

Equation (3.13) is known as traffic variability equations, which describes the approximate relationship among the inter-arrival time SCVs of all nodes.

Once arrival and service parameters $\left(\lambda_{a m}, C_{a m}^{2}, \tau_{m}, C_{s m}^{2}\right)$ at each node are available, one can utilize them to get performance estimates of the node. Steady-state performance equations for estimating waiting-time and queue-length related measures can be found in [1, 16-17].

\section{Backward Analysis Algorithm}

In the analysis of Section III, means and SCVs of interdeparture times are calculated for all network nodes based on given means and SCVs of the external arrival processes and nodal service processes, which we call a forward analysis procedure. Exploiting results of the forward analysis, we shall develop in this Section a backward analysis (BA), which calculates the desired means and SCVs of inter-arrival times for individual nodes given the specified output and nodal characteristics. These parameters may serve as references for monitoring and guiding the control of a re-entrant line.

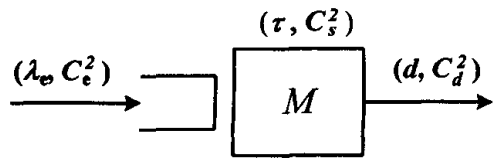

Figure 2: a $G I / G / I$ Queue
Let us first examine the very basic idea. Consider a $G I / G / 1$ queue as shown in Figure 2 with service time parameters $\left(\tau, C_{\mathrm{s}}{ }^{2}\right)$ and the desired mean output rate, $d$, and the upper bound of inter-departure time $\mathrm{SCV}, C_{d}^{2}$. From equation (3.11), one can easily calculate that the desired arrival rate

$$
\lambda_{e}=d
$$

and the SCV of inter-arrival time

$$
C_{e}^{2}=\frac{1}{1-\rho^{2}}\left(C_{d}^{2}-\rho^{2} C_{s}^{2}\right) \text {. }
$$

If the job arrival process is controlled to meet the target mean rate and to restrain its inter-arrival time SCV no greater than the value calculated in equation (4.1), then the output performance of the queue should meet the desired specifications.

Now we extend the basic idea described above to reentrant OQNs. To focus on our main concept without loss of generality in developing BA, we consider an aggregate OQN with the re-entrant structure as in Figure 1. Let $(d$, $C_{d}^{2}$ ) be the output specifications of the re-entrant OQN and $\left(\gamma_{1}, \gamma_{2}, \ldots, \gamma_{I}\right)$ be the given part-mix ratio of the output flow, where $\gamma_{1}+\gamma_{2}+\ldots+\gamma_{I}=1$.

\section{Desired Arrival Rates}

When the network is in a steady state, the desired external arrival rate should be equal to the desired output rate, i.e.,

$$
\begin{aligned}
& \lambda_{e}=d, \text { and } \\
& \lambda_{i}=\gamma_{i} \cdot d, \quad \text { for } i=1,2, \ldots, I .
\end{aligned}
$$

With Eqs. (4.2) and (3.2), the desired mean inter-arrival rate at each node, $\lambda_{a n}, n=1,2, \ldots, M$, can be calculated by solving the set of $M$ linear equations in (3.7). Service time parameters $\left(\tau_{m}, C_{s m}^{2}\right)$ and the traffic intensity (expected utilization) of node $m, \rho_{m}$, can then be calculated according to Eqs. (3.5), (3.6) and (3.8).

\section{Upper Bounds for Arrival Variability}

From equation (3.9), the relationship between $C_{d M}^{2}$, the SCV of node- $M$ departure process, and $C_{d}^{2}$, the SCV upper bound of the line output process, is

$$
C_{d}^{2}=q_{M O} C_{d M}^{2}+\left(1-q_{M O}\right) q_{M O} C_{a M}^{2}+\left(1-q_{M O}\right)^{2} C_{e M}^{2} .
$$

Departure process out of a node is effected by both the arrival and the service characteristics of the node. To express the inter-arrival time SCV of node $M$ in terms of its departure and service time parameters, we rewrite (4.3) as

$$
C_{d M}^{2}=\left[C_{d}^{2}-\left(1-q_{M o}\right) q_{M O} C_{a M}^{2}-\left(1-q_{M o}\right)^{2} C_{e M}^{2}\right] / q_{M O} .
$$

Applying equation (3.11) for $m=M$, we get

$$
C_{d M}^{2}=1+\left(1-\rho_{M}^{2}\right)\left(C_{a M}^{2}-1\right)+\frac{\rho_{M}^{2}\left(\max \left\{C_{s M}^{2}, 0.2\right\}-1\right)}{\sqrt{M_{M}}}
$$


Combine Eqs. (4.4) and (4.5) and derive

$$
C_{a M}^{2}=\frac{C_{d}^{2}-\left(1-q_{M O}\right)^{2} C_{e M}^{2}-q_{M O} \rho_{M}^{2}\left[1+\left(\max \left\{C_{S M}^{2}, 0.2\right\}-1\right) / \sqrt{M_{M}}\right]}{\Phi_{M O}\left[\left(1-q_{M O}\right)+\left(1-\rho_{M}^{2}\right)\right]} .
$$

Note that terms, except $C_{e M}^{2}$, in the right-hand side of Eq. (4.6) can all be directly calculated from parameters of output and machine service processes.

In the practice of semiconductor wafer fabrication, it is desirable that different types of wafers are loaded into a line with the same level of variability. Motivated by this fact, we assume equal SCVs for arrival processes of all part types, i.e., $C_{i}^{2}=C_{j}^{2}$ for $i \neq j$. It can then be easily derived from Eq. (3.4) that

$$
C_{e}^{2}=C_{i}^{2}, \forall i \text {, }
$$

and from Eq. (3.10) that

$$
C_{e m}^{2}=C_{e}^{2} \sum_{i=1}^{I}\left(\frac{\sum_{k=1}^{s_{i}} \lambda_{i} \mathbf{1}\left\{(i, k): m_{s_{i k}}=m\right\}}{\sum_{i=1}^{I} \sum_{k=1}^{s_{i}} \lambda_{i} \mathbf{1}\left\{(i, k): m_{s_{i k}}=m\right\}}\right)=C_{e}^{2}, \forall m .
$$

Let us substitute (4.8) into the traffic variability equation (Eq. (3.13)) and examine the relationship among the SCVs of nodal arrival processes. Eq. (3.13) then becomes

$$
C_{a n}^{2}=\alpha_{n}+\beta_{n} \cdot C_{e}^{2}+\sum_{m=1}^{M} C_{a m}^{2} b_{m n},
$$

where $\alpha_{n}=1-\varpi_{n}+\varpi_{n} \sum_{m=1}^{M}\left(\frac{\lambda_{m n}}{\lambda_{a n}}\right) q_{m n} \rho_{m}^{2} \chi_{m}$,

and $\quad \beta_{n}=\varpi_{n}\left(\frac{\lambda_{o n}}{\lambda_{a n}}\right)+\varpi_{n} \sum_{m=1}^{M}\left(\frac{\lambda_{m n}}{\lambda_{a n}}\right)\left(1-q_{m n}\right)^{2}$

$$
\text { for } n=1,2, \ldots, M \text {. }
$$

Eqs. (4.6) and (4.9) constitute a set of $M+1$ linear equations for $M+I$ variables. It can be expressed in a matrix form

$$
\left[\begin{array}{ccccc}
b_{11}-1 & b_{21} & \ldots & b_{M 1} & \beta_{1} \\
b_{12} & b_{22}-1 & \ldots & b_{M 2} & \beta_{2} \\
: & : & : & : & : \\
b_{1 M} & b_{2 M} & \ldots & b_{M M}-1 & \beta_{M} \\
0 & 0 & \ldots & -1 & \beta_{M+1}
\end{array}\right]\left[\begin{array}{c}
c_{a 1}^{2} \\
C_{d 2}^{2} \\
: \\
C_{a M}^{2} \\
C_{e}^{2}
\end{array}\right]+\left[\begin{array}{c}
\alpha_{1} \\
\alpha_{2} \\
: \\
\alpha_{M} \\
\alpha_{M+1}
\end{array}\right]=\mathbf{0}^{\prime}
$$

where

$$
\alpha_{M+1}=\frac{C_{d}^{2}-q_{M O} \rho_{M}^{2}\left[1+\left(\max \left\{C_{s M}^{2}, 0.2\right\}-1\right) / \sqrt{M_{M}}\right]}{q_{M O}\left[\left(1-q_{M O}\right)+\left(1-\rho_{M}^{2}\right)\right]},
$$

and

$$
\beta_{M+1}=\frac{-\left(1-q_{M O}\right)^{2}}{q_{M O}\left[\left(1-q_{M O}\right)+\left(1-\rho_{M}^{2}\right)\right]} .
$$

Parameters $C_{e}^{2}$ and $\left\{C_{a m}^{2}\right\}$ can be obtained by solving the set of linear equations (4.10).

\section{Preliminary Validation}

To preliminarily assess the validity of our BA algorithm, let us consider a simple re-entrant line with single part type, 2 different machines and 4 production steps as depicted in Figure 3. Table I gives the mean process time (MPT) and process time SCV for all steps. The output specifications are:

(1) desired throughput rate $\lambda_{d}=41$ wafer-per-hour;

(2) inter-departure time SCV $C_{d}^{2} \leq(1.088)^{2}$.

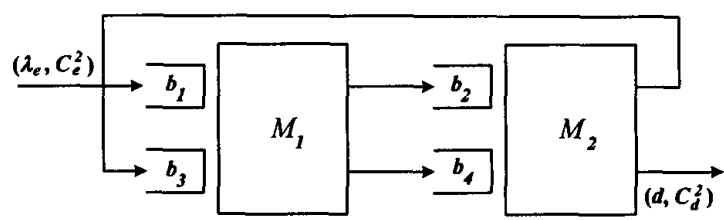

Figure 3: A 2-Machine 4-stage Re-entrant Line

Table I The Service Time Parameters

\begin{tabular}{|c|c|c|c|}
\hline Step & Machine Name & MPT (hour) & PT SCV \\
\hline 1 & $\mathrm{M}_{1}$ & $1 / 95$ & 1.0 \\
\hline 2 & $\mathrm{M}_{2}$ & $1 / 100$ & 1.5 \\
\hline 3 & $\mathrm{M}_{1}$ & $1 / 81$ & 1.0 \\
\hline 4 & $\mathrm{M}_{2}$ & $1 / 96$ & 1.5 \\
\hline
\end{tabular}

Our preliminary validation scheme first applies the BA procedure to the example; means and SCVs of inter-arrival times are derived. A simulation using the $\mathrm{Q}^{{ }^{\mathrm{TM}}}$ simulator [10] is then conducted over the example with the mean $\left(\lambda_{e}\right)$ and the $\operatorname{SCV}\left(C_{e}^{2}\right)$ of the external arrival process set as calculated by BA. Simulated results of internal and output departure process parameters are then compared with the calculated ones. They should be consistent if our BA is valid.

In our example, the elements of routing matrix are: $q_{11}=0$, $q_{12}=1, q_{20}=0.5, q_{21}=0.5$ and $q_{22}=0$. By applying BA, we obtain $C_{s 1}^{2}=1.013, C_{s 2}^{2}=1.501, \rho_{1}=0.938, \rho_{2}=$ $0.837, C_{a 1}{ }^{2}=1.091, C_{a 2}{ }^{2}=1.022$, and the external arrival variability parameter $C_{e}^{2}=0.999$. Mean cycle times of individual machine groups are then calculated [16-17]. Results listed in Table II are obtained by our software implementation of BA on a Pentium 100 personal computer in less than one second.

A simulation model is then constructed on top of the $\mathrm{Q}^{+\mathrm{TM}}$ simulator with the external arrival parameters $\left(\lambda_{e}, C_{e}^{2}\right)=$ $(41,1.0)$ and service time parameters set as those in Table I. Results of a seven million transactions simulation run are summarized in Table II. Results in Table II preliminarily suggest that BA is sufficiently accurate for quick estimate of performance requirements of a re-entrant 
Table II Simulation and BA Results

\begin{tabular}{|c|c|c|c|c|c|c|c|c|c|c|}
\cline { 2 - 11 } \multicolumn{1}{c|}{} & \multicolumn{4}{c|}{ Variability Parameters } & \multicolumn{3}{c|}{ Machine Cycle Time Performance } & \multicolumn{2}{c|}{ Depart. Process } \\
\cline { 2 - 11 } \multicolumn{1}{c|}{} & $C_{\mathrm{s} 1}{ }^{2}$ & $C_{\mathrm{s} 2}{ }^{2}$ & $C_{\mathrm{al}}{ }^{2}$ & $C_{\mathrm{a} 2}{ }^{2}$ & $\mathrm{E}\left[\mathrm{T}_{1}\right]$ & $\mathrm{V}\left[\mathrm{T}_{1}\right]$ & $\mathrm{E}\left[\mathrm{T}_{2}\right]$ & $\mathrm{V}\left[\mathrm{T}_{2}\right]$ & $\mathrm{E}[1 / d]$ & $C_{d}{ }^{2}$ \\
\hline $\mathrm{Q}^{+}$ & $(1.005)^{2}$ & $(1.225)^{2}$ & $(1.053)^{2}$ & $(1.016)^{2}$ & 0.188 & 0.034 & 0.075 & 0.007 & 0.024 & $(1.084)^{2}$ \\
\hline $\mathrm{BA}$ & $(1.006)^{2}$ & $(1.225)^{2}$ & $(1.045)^{2}$ & $(1.011)^{2}$ & 0.192 & 0.037 & 0.076 & 0.0064 & 0.0244 & $(1.088)^{2}$ \\
\hline
\end{tabular}

line and that if the external arrivals (jobs releasing) and internal arrivals (jobs dispatching) are controlled to match the performance requirements, output of the re-entrant line will then meet the specifications.

\section{Concluding Remarks}

In this paper, we developed, for re-entrant production lines, a method of translating inter-departure time mean and upper bounds on SCV of the output process to requirements for mean rates and upper bounds on SCV of individual machine input processes. A re-entrant production line was first modeled as an OQN. We combined decomposition-based queueing network analysis techniques to approximate, for the re-entrant $\mathrm{OQN}$, the relationship between the output specifications and the characteristics of external input and machine service processes. Due to the re-entrant feature, the inter-arrival time SCV of the external input process explicitly appears as a term of the inter-departure time SCV of the line output process. We then designed BA to derive, as two sets of linear equations, input flow requirements for machine groups from line output specifications and machine service characteristics. In our BA, FCFS is assumed as the service discipline for each node. In fact, service disciplines of individual nodes of a real re-entrant line are usually designed to result in better output performances than FCFS. So, the bounds on input processes obtained from BA by assuming FCFS intuitively may serve as upper bounds for real applications. Preliminary simulation study demonstrated the validity of our BA algorithm in its accuracy and computation speed. Further extension and application of BA to semiconductor fabrication lines are now being conducted. Results will be reported in the near future.

\section{References}

[1] G. R. Bitran and D. Tirupati, "Multiproduct queueing networks with deterministic routing: decomposition approach and the notion of interference," Management Science, vol. 34, pp.75-100, 1988.

[2] J. A. Buzacott and J. G. Shanthikumar, "Design of manufacturing systems using queueing models," Queueing Systems, vol. 12, pp. 135-213, 1992.

[3] H. Chen, J. M. Harrison, A. Mandelbaum, A. Van Ackere, and L. M. Wein "Empirical evaluation of a queueing network model for semiconductor wafer fabricationr," Operations Research, vol. 36, pp.202-
215, 1988.

[4] D. P. Connors, G. E. Feigin, and D. D. Yao, “A queueing network model for semiconductor manufacturing," IEEE Trans. Semi. Manuf., vol. 9, pp.412-427, 1996.

[5] J. E. Dayhoff and R. W. Atherton, "Simulation of VLSI manufacturing areas," VLSI Design., pp.84-92, 1984.

[6] J. E. Dayhoff and R. W. Atherton, "Signature analysis: simulation of inventory, cycle time and throughput tradeoffs in wafer fabrication," IEEE Trans. CHMT, vol. 9, pp.498-507, 1986.

[7] P. R. Kumar, "Re-entrant lines," Queueing Systems, vol. 13, pp. 87-110, 1993.

[8] S. Li, "Equi-variability graph approach for modeling of manufacturing systems," in Proc. $29^{\text {th }}$ Annu. Allerton Conf., Allerton, IL, pp.1063-1069, 1991.

[9] S. X. C. Lou and P. W. Kager, "A robust production control policy for VLSI wafer fabrication," IEEE Trans. Semi. Manuf., vol. 2, pp.159-164, 1989.

[10] B. Melamed, $Q^{+\mathrm{TM}}$ The AT\&T Performance Analysis Workstation, User Guide \& Reference Manual. AT\&T Bell Lab, Homdel, NJ., 1989.

[11] D. Meyersdorf, "Cycle time reduction," Lecture notes of Workshop on Layout Planning and Cycle Time Reduction for Semiconductor Manufacturing, HsinChu, Taiwan, 1996.

[12] L. Sattler, "Using queueing curve approximations in a fab to determine productivity improvements," Proceedings of the $7^{\text {th }}$ Annual IEEE/SEMI Advanced Semiconductor Manufacturing Conference and Workshop, Cambridge, MA, pp. 140-145, 1996.

[13] M. Segal and W. Whitt, "A queueing network analyzer for manufacturing," Pro. $12^{\text {th }}$ Intl. Teletraffic Conf., pp.1146-1152, 1989.

[14] T. E. Vollmann, W. L. Berry, and D. C. Whybark, Manufacturing Planning and Control Systems, IRWIN, 1992.

[15] F. Wang, "Consideration for manufacturing control systems," Key Note Speech Note of 1994 Semiconductor Manufacturing Workshop, Hsin-Chu, Taiwan, 1994.

[16] W. Whitt, "The queueing network analyzer," Bell System Technical Journal, vol. 62, pp. 2779-2815, 1983.

[17] W. Whitt, "Approximations for the GI/G/m queue," Production and Operations Management, vol. 2, pp.114-161, 1993. 